Draft Version OCtOBER 23, 2018

Preprint typeset using $\mathrm{IATEX}_{\mathrm{E}} \mathrm{X}$ style emulateapj v. 08/13/06

\title{
WIDE-FIELD INFRARED IMAGING POLARIMETRY OF THE NGC 6334 REGION: A NEST OF INFRARED REFLECTION NEBULAE
}

\author{
J. Hashimoto ${ }^{1}$, M. Tamura $^{1}$, R. Kandori ${ }^{1}$, N. Kusakabe ${ }^{1}$, Y. Nakajima ${ }^{1}$, \\ M. Kurita ${ }^{2}$, T. Nagata ${ }^{3}$, T. Nagayama ${ }^{3}$, J. Hough ${ }^{4}$, A. Chrysostomou ${ }^{5}$ \\ Draft version October 23, 2018
}

\begin{abstract}
We report the detection of eighteen infrared reflection nebulae (IRNe) in the $J, H, \& K s$ linear polarimetric observations of the NGC 6334 massive star-formation complex, of which 16 IRNe are new discoveries. Our images cover $\sim 180$ square arcminutes, one of the widest near-infrared polarization data in star-formation regions so far. These IRNe are most likely associated with embedded young OB stars at different evolutionary phases, showing a variety of sizes, morphologies, and polarization properties, which can be divided into four categories. We argue the different nebula characteristics to be a possible evolutionary sequence of circumstellar structures around young massive stars.

Subject headings: circumstellar matter — infrared: stars — polarization — ISM: individual (NGC 6334) — stars: formation
\end{abstract}

\section{INTRODUCTION}

Massive star-formation processes are poorly understood phenomena due to large distances, high extinction, and substantial source confusion. Recent infrared observations of (sub)arcsecond high-resolution allow one to study the complex nature of OB star-formations. In particular, near-infrared (NIR) imaging linear polarimetry is a powerful tool for investigating the circumstellar structures of the young stellar objects (YSOs). The radiation from YSOs still embedded in their parent molecular clouds is often scattered by the dust in their circumstellar structures, and is observed as infrared reflection nebula (IRN). This is also a good morphological tracer of mass outflow (Hodapp 1984; Sato et al. 1985), especially observed at high resolution (Tamura et al. 1991). In massive star-formation regions, NIR polarimetry can distinguish the IRNe from the H II region nebulae (Tamura et al. 2006), which allows one to easily uncover new IRNe buried in various emissions. In this Letter, we present wide-field linear polarization of the NGC 6334 region.

NGC 6334 is an active massive star-formation complex, a total bolometric luminosity $\sim 1.9 \times 10^{6} L_{\odot}$ (Loughran et al. 1986), at a distance of $1.7 \mathrm{kpc}$ (Neckel 1978). This giant $(\sim 10 \mathrm{pc})$ complex has several separated massive starformation sites. McBreen et al. (1979) show six strong far-infrared (FIR) sources named NGC 6334 I to VI (see Kraemer et al. (1999) for more details on other observations). Since each site is in the same parent cloud at the same distance, systematic studies of massive starfomation and its evolution are expected to be conducted.

\section{OBSERVATIONS \& DATA REDUCTION}

The NIR linear polarization images were obtained on June 25, 30, and July 12006 with the SIRIUS camera,

\footnotetext{
${ }^{1}$ National Astronomical Observatory, 2-21-1 Osawa, Mitaka Tokyo 181-8588; hashmtjn@optik.mtk.nao.ac.jp

2 Department of Astrophysics, Nagoya University, Chikusa-ku, Nagoya 464-8602

3 Department of Astronomy, Kyoto University, Sakyo-ku, Kyoto 606-8502

${ }^{4}$ Centre for Astrophysics Research, University of Hertfordshire, Hatfield HERTS AL10 9AB, UK

${ }^{5}$ Joint Astronomy Centre, 660 N. A'ohoku Place, Hilo, HI 96720
}

Simultaneous three-color InfraRed Imager for Unbiased Survey (Nagayama et al. 2003) and its polarimeter (SIRPOL; Kandori et al. 2006) mounted on the IRSF 1.4 $\mathrm{m}$ telescope at the South African Astronomical Observatory in Sutherland. The image scale of the array was $0^{\prime \prime} 45$ pixel $^{-1}$, giving a field of view of $7^{\prime} .7 \times 7^{\prime} .7$. The polarizations were measured by stepping the half waveplate to four angular positions $\left(0^{\circ}, 45^{\circ}, 22.5^{\circ}\right.$ and $\left.67.5^{\circ}\right)$. Ten dithered frames were observed per waveplate position, and we observed 9 sets for the object, giving $9 \times 10$ frames of $10 \mathrm{~s}$ integration per waveplate position. Seeing was $1 . .5$ (FWHM) in the $J$ band.

The data were reduced in the standard manner of infrared image reduction: subtracting a dark-frame and dividing by a flat-frame. In addition, the data for each waveplate position were registered, and then combined. The Stokes parameters were derived in the same manner as Tamura et al. (2006).

\section{RESULTS \& DISCUSSION}

The intensity images and linear polarization vector images in the NIR bands are shown in Figs.1(A) to (E). We briefly explain the detected IRNe in $\S 3.1$, which are selected from the highly polarized intensity and the polarization vector pattern, and then qualitatively discuss their polarization properties in $\S 3.2$.

\subsection{Discovery of Eighteen Infrared Reflection Nebulae}

NGC 6334 I. - We identify five IRNe as shown in Fig.1(A). IRN I-1 is illuminated by the ultracompact H II (UCH II) region of NGC $6334 \mathrm{~F}$ (Rodriguez et al. 1982). IRN I-2 and 3 are not illuminated by NGC $6334 \mathrm{~F}$ (Rodriguez et al. 1982) but are a monopolar and a bipolar nebula illuminated by DPT00 2 (De Buizer et al. 2002) and KDJ I-3 (Kraemer et al. 1999), respectively. IRN I-4 is also a bipolar nebula associated with an invisible NIR source. IRN I-5 around IRS I-14 (Straw et al. 1989) has a concentric polarization.

NGC 6334 II. - Fig.1(B) shows an approximately centrosymmetric polarized pattern. Though we calculate the position of the illuminating source from the intersection of the normals to the polarization vectors, marked 
with a $1 \sigma$ error circle in Fig.1(B), there are no bright sources within the error circle. Note that IRS II-22 (Straw et al. 1989) near the error circle is surrounded by a local concentric polarization. The edge of the spherical $\mathrm{H}$ II region has a higher $(\sim 5 \%)$ polarization than the vicinity of the error circle $(\sim 1 \%)$. We refer to this region as IRN II-1. Fig.1(B) also shows that an elongated IRN II-2, clearly seen in a polarized intensity image, has a polarization relatively well aligned with $\theta \sim 120^{\circ}$.

NGC 6334 III. - Similar to IRN II-1, Fig.1(C) shows a centrosymmetric polarized pattern on the edge of the $\mathrm{H}$ II region and the polarization is higher on the edge. Since IRS III-13 (Straw et al. 1989) is the brightest NIR source near the error circle, we believe it is the illuminating source. We refer to this region as IRN III-1. Another IRN is a relatively large bipolar nebula of IRN III-2 associated with a totally invisible source at NIR wavelengths.

NGC 6334 IV. — Fig.1(D) shows IRN IV-1, similar to IRN II-1 and III-1, has a centrosymmetric polarized pattern, and the positional error circle indicates that IRS IV-54 (Straw et al. 1989) is the illuminating source. IRN IV-2 and 3, better seen in a polarized intensity image, have relatively well aligned polarizations, $\theta \sim 170^{\circ}$ and $\sim 130^{\circ}$, respectively. There is IRN IV-4 with a concentric polarization around IRS IV-10 (Straw et al. 1989). IRN IV-5 is a bipolar nebula associated with an invisible source at NIR wavelengths. The faint IRN IV-6 is associated with an optically thin H II region, G351.24+0.65 (Moran et al. 1990).

NGC $6334 \mathrm{~V}$. - In spite of a bipolar morphology for IRN V-1 and 2 in Fig.1(E), Hashimoto et al. (2006) showed their illuminating sources are different (see also Chrysostomou et al. 1994). IRN V-3 is associated with a monopolar nebula with IRS V-41 (Straw et al. 1989).

\subsection{Classicifation and Possible Evolution of Infrared Reflection Nebulae}

Eighteen IRNe are detected in NGC 6334, and these IRNe can be divided into four categories of Type A to D. We conclude their illuminating sources are embedded or visible $\mathrm{OB}$ stars from the previous radio and infrared observations, except IRN I-4, II-1, 2, III-2, IV-3, and 5 without the previous radio and infrared observations (see Table 1 caption). Table 1 summarizes the list of eighteen IRNe and the nature of their illuminating sources.

The first category (Type A) contains IRNe with relatively well aligned polarization vectors. These IRNe have a non-point-like morphology and extend in a direction relatively perpendicular to polarization vectors. The illuminating sources are invisible at NIR wavelengths. The second category (Type B) contains monopolar/bipolar IRNe with several ten percent level polarization. The illuminating sources are invisible at NIR wavelengths. The third category (Type $C$ ) contains IRNe with concentric polarization around the visible illuminating sources at NIR wavelengths. These IRNe have a relatively small scale $(\lesssim 0.1 \mathrm{pc})$. The last category (Type $D)$ contains relatively large scale IRNe $(\gtrsim 1 \mathrm{pc})$. The edge of IRNe has centrosymmetric polarization and have a higher polarization than the vicinity of the illuminating source.

We argue the above classification can be placed along the approximate evolutionary sequence, i.e., Type $\mathrm{A}$ is the youngest and evolve into $\mathrm{D}$ through $\mathrm{B}$ and $\mathrm{C}$.

The reason we conclude Type $\mathrm{A}$ is the youngest is that the density of circumstellar material seems to be the highest. In this case, in contrast to the case of Type B described below, we expect that originally centrosymmetric polarization in the envelope region can be affected by multiple scattering, resulting in aligned vector patterns as observed (see e.g., Fischer et al. 1994). Whitney et al. (1997) in fact detected a similar aligned polarization in IRN associated with L1527 in Taurus. This IRN extends in the direction of the outflow (Tamura et al. 1996), perpendicular to polarization vectors. Zhang et al. (2007) recently found a compact redshifted outflow, whose extension is well correlated with IRN II-2. We also suggest that the illuminating source of IRN II-2 might be invisible at NIR wavelengths, and is therefore not likely to be IRS II-23 or 24. Similarly, the extensions of IRN IV-2 and 3 are relatively perpendicular to polarization vectors in each of the nebulae. Additionally, these IRNe are located in the vicinity of the submillimeter sources detected by Sandell (1999). Thus we also categorize them as Type A.

The more evolved Type B contains eight IRNe which are monopolar/bipolar nebulae. Since a close relationship has been suggested between IRNe and CO outflows (Nagata et al. 1984; Hodapp et al. 1984; Sato et al. 1985; Yamashita et al. 1989), we consider these IRNe correspond to the walls of the outflow material where the vectors are nearly centrosymmetric. Recently Arce et al. (2007) summarized the relation between the outflow collimation and its evolution; IRN III-2 is categorized as the most evolved one in their classification.

Small scale Type C contains IRN I-5 and IV-4 associated with the visible NIR sources. The transition from Type B to $\mathrm{C}$ we infer is as follows: the outflow associated with the Type B sources widens its opening angle with its evolution (Arce et al. 2007), then stellar radiation might be scattered by circumstellar material more concentric rather than bipolar/monopolar. Note that since it is possible that the concentric polarization is due to the inclination of outflow, these IRNe could be categorized as Type B.

Finally, Type D contains relatively large IRNe. The illuminating sources have well-developed H II regions that have had time to expand into the surrounding cloud. Due to stronger ionized gas emission in the vicinity of the illuminating sources, the degree of polarization is lower near the source than at the edge of the $\mathrm{H}$ II region. These IRNe could be due to reflection from dust at the ionization front.

In Orion, IRNe around IRc2 and $\mathrm{BN}$ correspond to Type B, and that around the Trapezium H II region is inferred to be Type D. In particular, that of BN is similar to IRN V-3 since the monopolar IRN is associated with the NIR visible illuminating source.

To summarize, we detected eighteen IRNe associated with young OB stars and suggest that the difference of the polarized patterns reflects the evolutionary sequence of IRNe; the youngest IRNe with well aligned polarization (Type A) evolve into monopolar/bipolar IRNe with roughly centrosymmetric polarization (Type B). When IRNe have concentric polarizations (Type $C$ ), the illuminating sources create $\mathrm{H}$ II regions, and then, IRNe become less distinct near the central sources with a centrosymmetric circular pattern at the region of the ionization boundary (Type $D$ ). We note, however, that care 
TABLE 1

Infrared REFLECtion Nebulae and their ILluminating Sources in NGC 6334

\begin{tabular}{|c|c|c|c|c|c|c|c|c|c|}
\hline \multirow[t]{2}{*}{ Type } & \multicolumn{2}{|l|}{$\operatorname{IRN}$} & \multicolumn{7}{|c|}{ Mluminating Source } \\
\hline & Name & $\begin{array}{l}\text { Size } \\
\text { (pc) }\end{array}$ & $\begin{array}{l}\text { Position } \\
(\mathrm{h}: \mathrm{m}: \mathrm{s})\end{array}$ & $\begin{array}{c}\mathrm{J} 2000)^{\mathrm{a}} \\
\left({ }^{\circ}:{ }^{\prime}:{ }^{\prime \prime}\right)\end{array}$ & Identification $^{b}$ & $\begin{array}{l}\text { Spitzer } \\
8.0 \mu \mathrm{m}\end{array}$ & Maser $^{\mathrm{C}}$ & $\begin{array}{c}K \text { band }^{\mathrm{d}} \\
(\mathrm{mag})\end{array}$ & ZAMS Sp. $^{\mathrm{e}}$ \\
\hline \multirow{3}{*}{ A } & IRN II-2 & 0.5 & {[} & $\overline{-}$ & invisible & No & - & - & unknown \\
\hline & IRN IV-2 & 0.05 & 172017.85 & -355453.0 & KDJ IV-3 (MIR), MM2 & No & - & - & B1 \\
\hline & IRN IV-3 & 0.1 & 172023.82 & -355456.3 & MM3? & Yes & $\mathrm{OH}$ & - & unknown \\
\hline \multirow{8}{*}{ B } & IRN I-2 & 0.1 & 172053.77 & -354700.6 & DPT00 2 (MIR) & No & - & - & B4 \\
\hline & IRN I-3 & 0.2 & 172054.59 & -354702.6 & KDJ I-3 (MIR), IRS I-9 (NIR) & No & — & 12.00 & B0 \\
\hline & IRN I-4 & 0.2 & 172051.90 & $\begin{array}{llll}-35 & 47 & 04.2\end{array}$ & invisible & Yes & — & — & unknown \\
\hline & IRN III-2 & 0.5 & 172034.24 & -355047.9 & invisible & Yes & — & - & unknown \\
\hline & IRN IV-5 & 0.5 & 172015.35 & $\begin{array}{lll}-35 & 56 & 04.7\end{array}$ & invisible & No & — & - & unknown \\
\hline & IRN V-1 & 0.4 & 171957.79 & -355750.8 & WN-A1 (NIR) & No & $\mathrm{NH}_{3}, \mathrm{OH}$ & — & B \\
\hline & IRN V-2 & 0.4 & 171957.46 & -355752.5 & KDJ V-4 (MIR) & Yes & $\mathrm{NH}_{3}$ & — & B2 \\
\hline & IRN V-3 & 0.15 & 172000.35 & $-35 \quad 57 \quad 17.9$ & IRS V-41 (NIR) & Yes & — & 8.50 & late $\mathrm{O}$ \\
\hline \multirow[t]{2}{*}{$\mathrm{C}$} & IRN I-5 & 0.15 & 172055.08 & -354645.0 & IRS I-14 (NIR) & Yes & $\mathrm{NH}_{3}$ & 11.56 & early B \\
\hline & IRN IV-4 & 0.15 & 172020.72 & $\begin{array}{lll}-35 & 55 & 04.7\end{array}$ & IRS IV-10 (NIR) & Yes & - & 9.76 & late $\mathrm{O}$ \\
\hline \multirow{5}{*}{$\mathrm{D}$} & IRN I-1 & 0.3 & 172053.50 & -354702.8 & NGC 6334 F, IRS I-10 (NIR) & Yes & $\mathrm{OH}$ & 10.24 & B0 \\
\hline & IRN II-1 & 2.0 & 172046.93 & -354905.4 & invisible & No & — & - & unknown \\
\hline & IRN III-1 & 1.0 & 172031.78 & $-3551 \quad 11.4$ & NGC 6334 C, IRS III-13 (NIR) & No & - & 7.37 & O8 \\
\hline & IRN IV-1 & 0.6 & 172018.62 & -355405.1 & IRS IV-54 (NIR) & Yes & - & 9.87 & late $\mathrm{O}$ \\
\hline & IRN IV-6 & 0.1 & 172022.62 & $-35 \quad 5519.9$ & G351.24+0.65 (radio) & No & - & - & B0.5 \\
\hline
\end{tabular}

a The positions for the invisible or unkown illuminating sources of IRN I-4, II-1, III-2, and IV-5 are derived by our polarization data; IRN IV-2 is from Kraemer et al. (1999), IRN IV-3 is from the Spitzer GLIMPSEII survey, IRN I-2 and 3 are from De Buizer et al. (2002), IRN V-1 and 2 are from Hashimoto et al. (2006), IRN IV-6 is from Balser et al. (2001), the invisible illuminating sources of IRN II-2 cannot be well determined from our data, and the other visible illuminating sources at NIR wavelengths are from 2MASS-PSC.

b Identifications are performed with IR and radio sources; KDJ I-3 and IV-3 are from Kraemer et al. (1999), MM2 and 3 are from Sandell (1999), DPT00 2 is from De Buizer et al. (2002), WN-A1 and KDJ V-4 are from Hashimoto et al. (2006), NGC 6334 C and F are from Rodriguez et al. (1982), G351.24+0.65 is from Moran et al. (1990), and the other NIR sources are from Straw et al. (1989). "invisible" means invisible at NIR wavelengths.

c From Brooks \& Whiteoak (2001) and Kraemer et al. (1999).

d The $K$ band magnitude of the sources are from Straw et al. (1989).

e The zero-age main-sequence (ZAMS) spectral type for the illuminating sources of each IRN; IRN IV-2 is from Jackson \& Kraemer (1999), IRN I-2 is from De Buizer et al. (2002), IRN I-3 is from Persi et al. (1998), IRN V-1 and 2 are from Hashimoto et al. (2006), IRN I-5, IV-1, IV-4, and V-3 are from Straw et al. (1989), IRN I-1 and III-1 are from Rodriguez et al. (1982), and IRN IV-6 is from Balser et al. (2001). Since the IR/radio data are not available, the illuminating source of IRN I-4, II-1, 2, III-2, IV-3 and 5 are unknown.

must be taken to infer above conclusions from the polarization pattern alone. Further high resolution radio observations for kinematical information should be undertaken to verify our IRN evolutionary hypothesis.

We are grateful to an anonymous referee for providing

\section{REFERENCES}

Arce, H. G., Shepherd, D., Gueth, F., Lee, C. F., Bachiller, R. Rosen, A., \& Beuther, H. 2007, in Protostars and Planets V, ed. B. Reipurth, D. Jewitt, \& K. Keil (Tucson: Univ. Arizona Press), 245

Brooks, K. J., \& Whiteoak, J. B. 2001, MNRAS, 320, 465

Balser, D. S., Goss, W. M., \& De Pree, C. G. 2001, ApJ, 121, 371.

Chrysostomou, A., Hough, J. H., Aspin, C., \& Bailey, J. A. 1994, MNRAS, 268, L63

De Buizer, J. M., Radomski, J. T., Pina, R. K., \& Telesco, C. M. 2002, ApJ, 580, 305

Fischer, O., Henning, Th., \& Yorke, H. W. 1994, A\&A, 284, 187

Hashimoto, J., et al. 2006, PASJ, 59, 221

Hodapp, K. W. 1984, A\&A, 141, 255

Jackson, J. M., \& Kraemer, K. E. 1999, ApJ, 512, 260

Kraemer, K. E., Deutsch, L. K., Jackson, J. M., Hoffmanm, W., Hora, J., Dayal, A., \& Fazio, G. 1999, ApJ, 516, 817

Kandori, R. et al. 2006, Proc. SPIE, 6269, 159.

Loughran, L., McBreen, B., Fazio, G. G., Rangarajan, T. N., Maxson, C. W., Serio, S., Sciortino, S., \& Ray, T. P. 1986, ApJ, 303,629

Mcbreen, B., Fazio, G. G., Stier, M., \& Wright, E. L. 1979, ApJ, 232, L183

Moran, J. M., Rodriguez, L. F., Greene, B., \& Backer, D. C. 1990, ApJ, 348, 147 many useful comments leading to an improved paper. This research was partly supported by Grants-in-Aid for Scientific Research on Priority Areas from the Ministry of Education, Culture, Sports, Science and Technology (MEXT) and No.16340061, 16077204, and 16340061.
Neckel, T. 1978, A\&A, 69, 51

Nagayama, T., et al. 2003, Proc. SPIE, 4841, 459.

Nagata, T., Sato, S., \& Kobayashi, Y. 1983, A\&A, 119, L1.

Persi, P., Tapia, M., Felli, M., Lagage, P. O., \& Ferrari-Toniolo, M. 1998, A\&A, 336, 1024

Rodriguez, L. F., Canto, J., \& Moran, J. M. 1982, ApJ, 255, 103

Sandell, G. 1999, A\&A, 343, 281

Straw, S. M., Hyland, A. R., \& Mcgregor, P. J. 1989, ApJS, 69, 99. Sato, S., Nagata, T., Nishida, M., Tanaka, M., \& Yamashita, T. 1985, ApJ, 291, 708

Tamura, M., Gatley, I., Joyce, R. R., Ueno, M., Suto, H., \& Sekiguchi, M. 1991, ApJ, 378, 611

Tamura, M., Ohashi, N., Hirano, N., Itho, Y., \& MoriartySchieven, G. H. 1996, AJ, 112, 2076

Tamura, M. et al. 2006, ApJ, 649, L29

Whitney, B. A., Kenyon, S. J., \& Gomez, M. 1997, ApJ, 485, 703 Yamashita, T., Suzuki, H., Kaifu, N., Tamura, M., Mountain, C. M., \& Moore, T. J. T. 1989, ApJ, 347, 894.

Zhang, J. S., Henkel, C., Mauersberger, R., Chin, Y. N., Menten, K. M., Tieftrunk, A. R, \& Belloche, A. 2007, A\&A, 465,887 

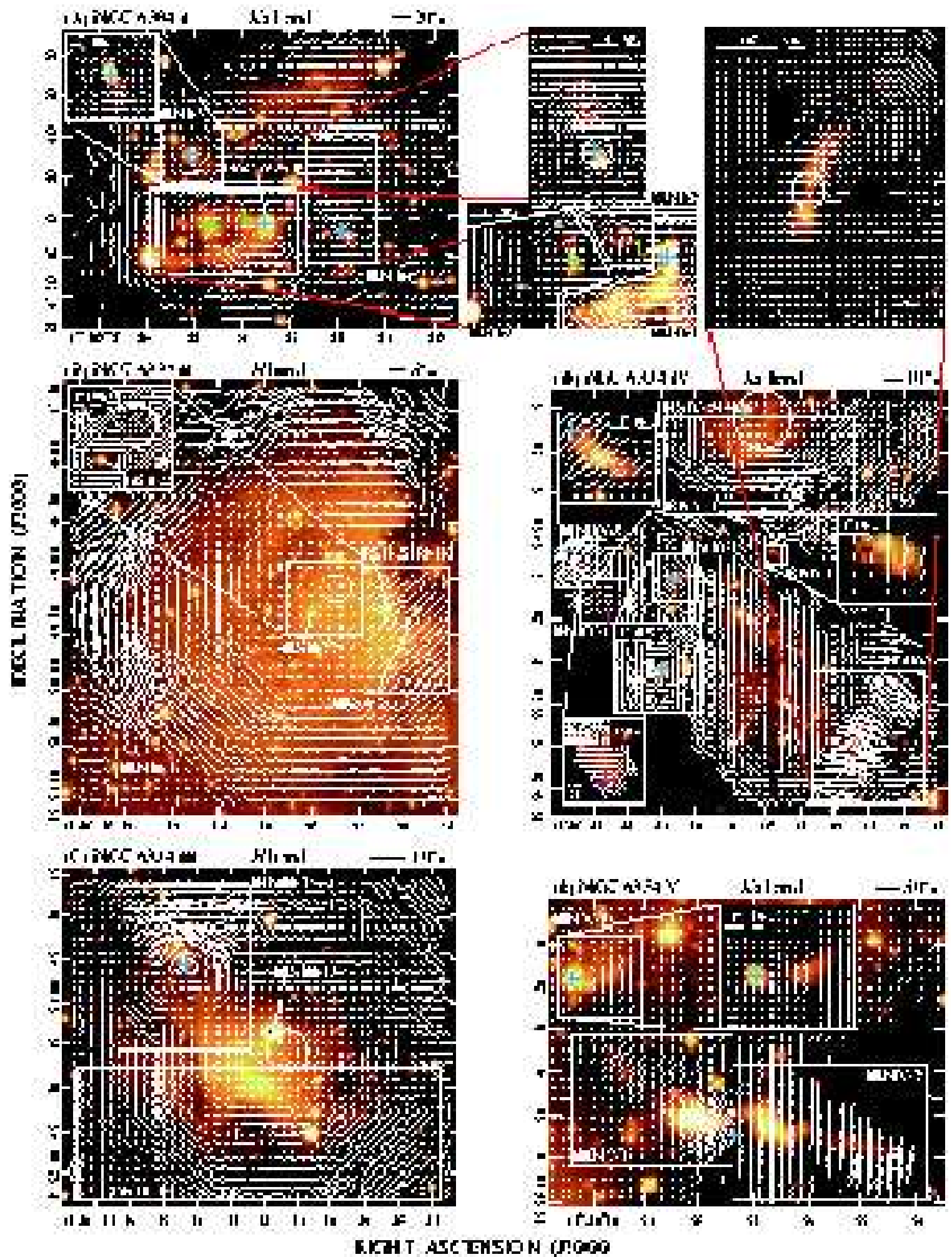

FIG. 1.- Polarization vectors are superposed on the intensity images in the $H$ or $K s$ band. In the magnifications, except for IRN IV-6, the vectors are superposed on the polarized intensity images in the same band. The white square indicates each IRN, and the circle shows each position of the illuminating sources with $1 \sigma$ error. Blue crosses represent the positions of Spitzer $8.0 \mu \mathrm{m}$ sources, green crosses represent the MIR sources for IRN I-2 and 3, and purple cross represents the radio source for IRN IV-6. For IRN I-1, I-5, IV-4, and V-3, since illuminating sources are identified with NIR sources, the error circles are not marked. 